Methods We randomised consecutive patients diagnosed with ASUC ( modified Truelove and Witt's classification) to receive placebo infusions or combination antibiotics (intravenous ceftriaxone and metronidazole) groups. Response as defined by oxford criteria was used to assess response on day three. We also assessed changes in partial Mayo score, CRP levels and reduction in fecal calprotectin at day three. Also, we assessed the need for second line drug therapy, colectomy, length of hospital stay and mortality by day 28 .

Results Fifty patients were randomised: 25 in each arm (Median age: 33, IQR 25-45, 23(46\%) males). Twenty-two patients had extensive disease, while the median disease duration was 24 months. Sixteen patients (64\%)in antibiotic arm responded (complete and partial response) at day three while $18(72 \%)$ in the placebo arm responded. Three patients from the antibiotic group underwent colectomy. Three patients in the antibiotic arm received intravenous cyclosporine, whereas four patients in the placebo group received cyclosporine $(p=0.725)$. There was no significant difference in change in CRP, Partial Mayo and fecal calprotectin between the two groups on day three.

Conclusions Combination of intravenous ceftriaxone and metronidazole in patients with ASUC neither improved the day 3 response nor reduced the need for second line therapy.

\section{IDDF2020-ABS-0146 USE OF ANTIBIOTICS IN ULCERATIVE COLITIS: SYSTEMATIC REVIEW AND META- ANALYSIS}

DayaKrishna Jha*, Shubhra Mishra, Anupam K Singh, Praveen Kumar-M, Amol Patil, Vishal Sharma. Postgraduate Institute of Medical Education and Research, India

\subsection{6/gutjnl-2020-IDDF.92}

Background Antibiotics have a proven role in the induction of remission in Crohn disease and treatment of pouchitis. However, the role of antibiotics for disease activity and remission maintainence in ulcerative colitis (UC) is uncertain.

Methods We searched electronic databases (Pubmed, Embase and CENTRAL) for randomised trials using various search terms to identify studies reporting on the use of antibiotics for induction or maintainence of remission in ulcerative colitis. The outcomes assessed were a clinical response, need for second line therapy, colectomy and adverse effects. Subgroup analysis to clarify the mode of administration (oral or intravenous), number of antibiotics (single or combination) or the setting of use (acute severe UC or active non-severe UC) were also done.

Results Eventually, 13 trials including 785 patients were included. The pooled odds ratio of achieving clinical response with antibiotic use was 1.74 (95\% CI, 1.17- 2.58). No differences were noted in the relapse rates, need for second line therapy, colectomy or adverse effects with the use of antibiotics. Subgroup analysis showed no differences with use of single or combination of antibiotics. The use of oral antibiotics in the setting of non-severe active UC could have some benefit in clinical response. No benefit of intravenous antibiotics in setting of acute severe ulcerative colitis was noted. The included studies were heterogeneous because of different included populations, type and duration of antibiotics use and differences in definitions and time of assessment of clinical response.
Conclusions The results suggest a lack of benefit of intravenous antibiotics in the setting of acute severe UC. The use of oral antibiotics for induction of maintenance needs further evaluation

\section{IDDF2020-ABS-0147 DEVELOPMENT OF A VALIDATED NOMOGRAM TO PREDICT AGGRESSIVE CROHN'S DISEASE: A RETROSPECTIVE COHORT STUDY}

${ }^{1} J$ iayin Yao*, 'Junzhang Zhao, ${ }^{2}$ Bang Hu, ${ }^{1}$ Min Zhi. 'Department of Gastroenterology, Guangdong Provincial Key Laboratory of Colorectal and Pelvic Floor Disease, The Sixth Affiliated Hospital of Sun Yat-Sen University, China; ${ }^{2}$ Department of Colorectal Surgery, Guangdong Provincial Key Laboratory of Colorectal and Pelvic Floor Disease, The Sixth Affiliated Hospital of Sun Yat-Sen University, China

\subsection{6/gutjnl-2020-IDDF.93}

Background Predicting aggressive Crohn's disease (CD) is crucial for determining therapeutic strategies. We aimed to develop a prognostic model to predict disease-related complications leading to early-onset surgery within 1 year after diagnosis of $\mathrm{CD}$ and to create a nomogram to facilitate clinical decision-making.

Methods This retrospective study was conducted from January 1, 2012, to December 31, 2016, in a single tertiary referral center, using data from patients newly diagnosed with $\mathrm{CD}$ and showing B1 behavior according to Montreal classification. The model was established using multivariable logistic regression analysis with evaluation of the receiver operating characteristic (ROC) curves and areas under the curve (AUC). The model was calibrated and assessed for discrimination. Further, a userfriendly nomogram was created.

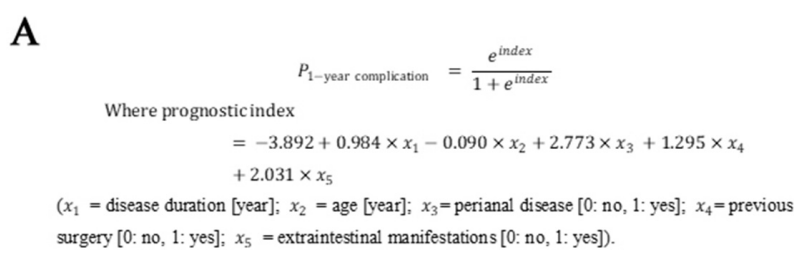

Abstract IDDF2020-ABS-0147 Figure 1A

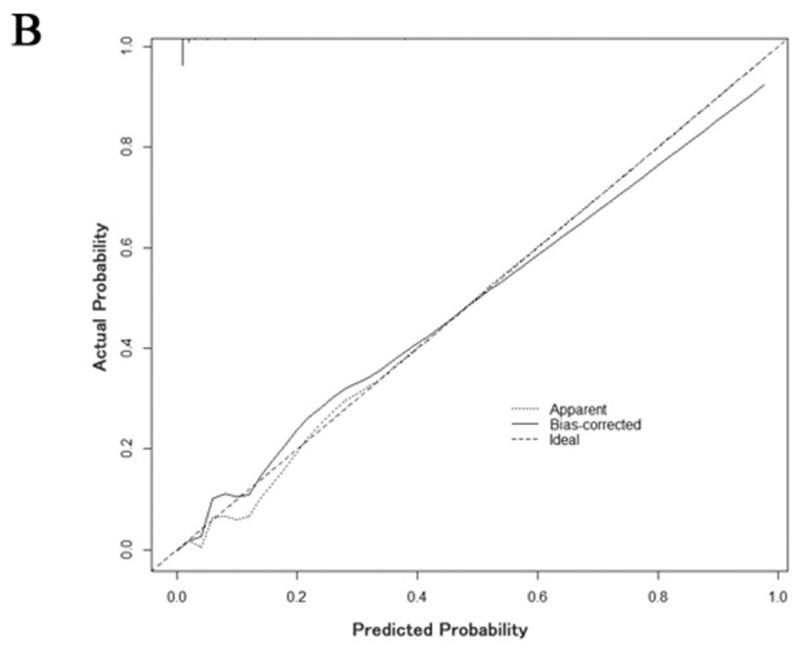

Abstract IDDF2020-ABS-0147 Figure 1B 